\section{Generalisierte Angst}

Nach den Ergebnissen einer aktuellen Studie eignet sich der KalziumkanalModulator Pregabalin (Lyrica ${ }^{\varpi}$ ) neben dem bisher etablierten Einsatz in der Monotherapie auch als Add-on-Therapie bei therapierefraktären Patienten mit Generalisierter Angststörung (GAD), die nur partiell auf andere First-Line-Präparate (SSRI, SNRI) ansprechen [Rickels K et al. Int Clin Psychopharmacol 2012; 27:142 - 50]. In der achtwöchigen Open-Label-Phase der doppelblinden, placebokontrollierten Studie erhielten GAD-Patienten Paroxetin, Escitalopram oder Venlafaxin. Die Patienten, die trotz zwei Therapieversuchen mit SSRI oder SNRI nur partiell auf das jeweilige Medikament reagiert hatten (HAM-A-Wert $\geq 16$ ), wurden randomisiert $(\mathrm{n}=356)$. $\mathrm{Zu}$ sätzlich zur bereits bestehenden Therapie erhielten 180 Patienten Pregabalin (150-600 mg/d), 176 Patienten Placebo. Primärer Endpunkt war die Veränderung auf der Hamilton Anxiety Rating Scale (HAM-A). Zu Beginn der Studie betrugen die mittleren HAM-A-Werte der Kohorte 20,7 beziehungsweise 21,4. Nach acht Wochen hatte sich der HAM-A-Wert in der Pregabalin-Gruppe signifikant gegenüber der PlaceboGruppe verbessert (-7,4 vs. $-6,4, \mathrm{p}<$ 0,05). Unter der Begleittherapie mit Pregabalin waren zudem die Ansprechraten signifikant höher als in der Placebo-Gruppe ( $47,5 \%$ vs. $35,2 \%$, p = $0,0145)$, wobei eine $50 \%$ ige Reduktion des HAM-A als Response galt. red

Pfizer

\section{Interaktive Fortbildung}

Begeben Sie sich in einer interaktiven Online-Fortbildung zusammen mit dem Medizindetektiv Dr. Wolgemuth auf Spurensuche: Julia Müller war lebenslustig und erfolgreich, bis sie durch eine Depression antriebslos wurde und Rückenschmerzen und Beklemmungen in der Brust bekam. Die Behandlung mit einem Trizyklikum änderte wenig und führte zu Müdigkeit und Gewichtszunahme. Lösen Sie den Fall unter www.lillyfortbildung.de.. therapiert werden, lässt sich durch eine Kombination mit Memantine die klinische Verschlechterung wirksamer hinauszögern als durch Monotherapie mit dem AChE-Hemmer alleine. Das belegen nach Angaben von Riepe die Responderanalysen der gepoolten Daten von $592 \mathrm{~Pa}$ tienten (MMSE < 20), die entweder die Kombination oder Donepezil plus Placebo erhalten hatten. Im Verlauf von sechs Monaten kam es bei 19,6\% der Patienten unter Donepezil-Monotherapie zu einer ausgeprägten klinischen Verschlechterung, aber nur bei $8 \%$ der Patienten unter der Kombinationstherapie. Für jegli- che klinische Verschlechterung betrugen die Werte 17,9\% und 28,5\% (Kombinations-/Monotherapie). „Eine generelle Empfehlung für die Kombinationstherapie zu geben, wäre derzeit nicht leitliniengemäß“, sagte Riepe, es sei eher eine mögliche künftige Option, Antidementiva mit verschiedenen Wirkprinzipien primär zu kombinieren.

Dr. Nicola Siegmund-Schultze, freie Medizinjournalistin

Alzheimer Demenz - eine interdisziplinäre Herausforderung; 14.9.2012 in Bonn Veranstalter: Merz

Therapie der Multiplen Sklerose

\title{
Eskalation bei entsprechender Indikation so früh wie möglich
}

Eineinhalb Jahre nach der Zulassung betonten Experten auf einer Pressekonferenz im Rahmen des DGN-Kongresses in Hamburg, dass bei Indikation für eine Eskalation die Datenlage dafür spreche, das erste orale MS-Therapeutikum Fingolimod (Gilenya ${ }^{\circledast}$ ) so früh wie möglich einzusetzen.

Grundsätzliches Ziel der MS-Therapie sei es, die subklinische Krankheitsaktivität zu reduzieren und den Patienten möglichst vor Schüben zu schützen. Zeige die MS unter einer immunmodulatorischen Basistherapie Krankheitsaktivität sei eine frühe Eskalationstherapie zum Beispiel mit Fingolimod zu erwägen, sagte Professor Dr. Volker Limmroth, Ärztlicher Direktor der Klinik für Neurologie und Palliativmedizin Köln-Merheim.

\section{Initialer Vorsprung nicht aufgeholt}

So sprächen unter anderem die Daten der FREEDOMS-Extensionsstudie dafür, das darin aufgezeigte „Window of Opportunity“ zu nutzen und früh mit einer Eskalationstherapie wie Fingolimod zu beginnen. Denn während der zweijährigen doppelblinden Kernstudie war die jährliche Schubrate unter Fingolimod gegenüber Placebo um $54 \%$ reduziert ( 1,8 vs. $0,4 ; \mathrm{p}<0,001)$. Im offenen Follow-up blieb sie konstant auf diesem niedrigen Niveau. Zwar ging die jährliche Schubrate auch nach Umstellung von Placebo auf Fingolimod signifikant um 55\% zurück $(\mathrm{p}<0,001)$, kontinuierlich mit Fingoli- mod behandelte Patienten waren am Ende mit $74 \%$ jedoch signifikant häufiger ohne bestätigte Behinderungsprogression als jene der Switch-Gruppe mit 66\% (p $=0,017)$. Der Anteil der nach vier Jahren schubfreien Patienten war ebenfalls signifikant höher (59 vs. 37\%; p < 0,001). Den durch die initiale Fingolimod-Therapie erzielten Vorsprung holten die erst nach zwei Jahren umgestellten Patienten somit nicht wieder auf.

Eine „frühe“ Umstellung bedeute dabei, Fingolimod einzusetzen, wenn nach einjähriger Therapie mit einem Beta-Interferon mindestens ein Schub aufgetreten sei und im kranialen MRT neun oder mehr hyperintense Läsionen oder mindestens eine Gadolinium-anreichernde Läsion sichtbar seien. Aber auch bei Patienten mit gleichbleibender Schubrate oder anhaltend schweren Schüben trotz Beta-Interferon-Therapie sei eine Eskalation sinnvoll. Limmroth verwies in diesem Zusammenhang auf die Leitlinien, die erst unlängst von der DGN und dem Krankheitsbezogenen Kompetenznetz Multiple Sklerose (KKNMS) aktualisiert worden sind.

Dr. Gunter Freese, Springer Medizin

\footnotetext{
Pressegespräch „MS-Therapie: Jeder Schub ist einer zu viel - besser frühzeitig eskalieren"; im Rahmen des 84. DGN-Kongresses, Hamburg, 26.9.2012; Veranstalter: Novartis
} 